FSHD to chromosome 4 will help to clarify some of the counseling issues, including families diagnosed as FSH type spinal muscular atrophy where the same genetic locus is involved. The risk of disability and wheelchair requirement in later life can be assessed by the reported age of onset and the occurrence of proximal lower limb weakness by age 20 years. The authors estimate that $19 \%$ of FSHD heteroxygotes will require a wheelchair by 40 years or over and $30 \%$ remain only mildly affected throughout life. Since asymmetry of weakness correlates with handedness, the overuse of limbs and particularly body building exercises should be discouraged.

\title{
HEAD CIRCUMFERENCE AND IQ IN DUCHENNE MD
}

The head circumferences of 64 patients with Duchenne muscular dystrophy were greater than normal and the intellectual performance tested by the Wechsler was significantly impaired in a study performed at The Royal Liverpool Children's Hospital, Alder Hey, Liverpool and The University of Newcastle upon Tyne. There was no correlation between head circumference and intellectual performance. Subsequent studies in 19 patients monitored with CT suggested that the large head was related to increased brain size (Appleton RE et al. Head circumference and intellectual performance of patients with Duchenne muscular dystrophy. Dev Med Child Neurol Oct $1991 ; \underline{33}: 884-890)$.

COMMENT. These patients with DMD appear to have relative and, less frequently, absolute macrocephaly which was unrelated to height and showed some familial tendency, the fathers having larger heads. The cause of the macrocephaly and increased brain size was not determined. Macrocephaly was defined as relative if the head circumference was disproportionately large for height or absolute, if greater than the 97 th centile. There was no correlation between the head circumference and intellectual performance of either the entire group of 47 patients or of the 12 patients with absolute macrocephaly.

The predictive value of a reduction in the size of the brain in the first year for mental retardation at 7 years has been investigated in 41 term infants with microcephaly. Half the microcephalic children were mentally retarded at the age of 7 . Head size 2 standard deviations below the mean had a very low predictive value for mental retardation (11\%). Disproportionate head-to-height ratio did not significantly affect outcome (Dolk H. Dev Med Child Neurol Nov 1991; 33:974983). The study was based on the US National Collaborative Perinatal Project Data of over 50,000 pregnancies and was performed at the Department of Epidemiology, Catholic University of Louvain, Brussels, Belgium.

\section{DYSTROPHIN IN LIMB-GIRDLE DYSTROPHY}

Dystrophin content in muscle was analyzed by both immunofluorescence and immunoblot in 41 patients with a clinical diagnosis of limb-girdle muscular dystrophy seen at the National Institute of Neuroscience, Tokyo, 